\title{
A comparative study of hyperspectral unmixing using different algorithm approaches
}

\author{
Ain Zat Mohd Yusof, Redzuan Abdul Manap, Abd Majid Darsono \\ Centre of Telecommunication Research and Innovation (CeTRI), Faculty of Electronics and Computer Engineering, \\ Universiti Teknikal Malaysia Melaka (UTeM), Malaysia.
}

\begin{tabular}{|c|c|}
\hline Article Info & ABSTRACT \\
\hline Article history: & \multirow{9}{*}{$\begin{array}{l}\text { Hyperspectral unmixing (HU) is an important technique for remotely sensed } \\
\text { hyperspectral data exploitation. Hyperspectral unmixing is required to get } \\
\text { an accurate estimation due to low spatial resolution of hyperspectral cameras, } \\
\text { microscopic material mixing, and multiple scattering that cause spectra } \\
\text { measured by hyperspectral cameras are mixtures of spectra of materials in } \\
\text { a scene. It is a process of estimating constituent endmembers and their } \\
\text { fractional abundances present at each pixel in hyperspectral image. } \\
\text { Researchers have devised and investigated many models searching for } \\
\text { robust, stable, tractable and accurate unmixing algorithm. Such algorithm are } \\
\text { highly desirable to avoid propagation of errors within the process. This paper } \\
\text { presents the comparison of hyperspectral unmixing method by using different } \\
\text { kind of algorithms. These algorithms are named VCA, NFINDR, SISAL, and } \\
\text { CoNMF. The performance of unmixing process is evaluated by calculating } \\
\text { the SAD (spectral angle distance) for each endmembers by using same input } \\
\text { of hyperspectral data for different algorithm. }\end{array}$} \\
\hline Received Feb 14, 2020 & \\
\hline Revised Apr 11, 2020 & \\
\hline Accepted Apr 27, 2020 & \\
\hline Keywords: & \\
\hline Hyperspectral cameras & \\
\hline Hyperspectral unmixing & \\
\hline Spectral angle distance & \\
\hline & \\
\hline
\end{tabular}

Copyright $(0) 2020$ Institute of Advanced Engineering and Science. All rights reserved.

\section{Corresponding Author:}

Redzuan Abdul Manap,

Centre of Telecommunication Research and Innovation (CeTRI),

Faculty of Electronics and Computer Engineering,

Universiti Teknikal Malaysia Melaka (UTeM),

Hang Tuah Jaya, Durian Tunggal 76100 Melaka, Malaysia.

Email: redzuan@utem.edu.my

\section{INTRODUCTION}

The imaging concept is divided into two types which are multispectral and hyperspectral. It collects and processes the information from across the electromagnetic spectrum [1]. This concept enables capture of an image simultaneously in hundreds of narrow continuous spectral band. The advantage is it provides a large amount of data including of the complete spectrum of ground object due to problem of resolution limitation of the sensors and the variability of the ground surface. The observation of one pixel may contain several disparate substances causing it to be a "mixed pixel". Furthermore, to utilize the hyperspectral information, these mixed pixel must be decomposed into a set of constituent spectra called endmember signatures and their corresponding proportions called abundances [1,2]. Keeping in mind the end goal to make full utilization of the information, hyperspectral unmixing (HU) has becomes an essential produces, which deteriorates a mixed pixel into a gathering on constituent materials additionally called endmember and their relative proportions. HU alludes to any procedure that isolates the pixel spectra from a hyperspectral picture into a collection of constituent spectra, or spectral signatures, called endmembers and a set of fractional abundances, one set for each pixel [3]. The endmembers are for the most part expected to represent the pure materials present in the images and the set of abundances, or simply abundances at every pixel to represent to the level of each endmember that is available in the pixel. Over the last years, different HU algorithms are developed for each of the three main steps of the spectral unmixing chain [4]: 
1) estimation of the number of endmembers in a scene; 2) identification of the spectral signatures of the endmembers; and 3) estimation of the fractional abundance of each endmember in each pixel of the scene. However, few algorithms can perform all the stages involved in the hyperspectral unmixing process. Such algorithms are highly desirable to avoid the propagation of errors within the chain [4]. For example, a signal unmixing methodology to extract pure vegetation signal from individual mixed pixel of scene consisting of soil and vegetation is proposed in [5]. In this paper, we study several hyperspectral unmixing (HU) algorithms, namely NFINDR [6, 7], VCA [8, 9], SISAL [10, 11], and CoNMF [12, 13]. The performance of their unmixing process is evaluated by calculating the spectral angle distance (SAD) for each endmembers using the same input of hyperspectral data $[14,15]$. However, there is still no in-depth comparative study have been conducted for these well-known methods in hyperspectal unmixing process. So, the research will determine the performance of current unmixing method that can help with reliable detection and identification. It will give benefit to country especially in agriculture production that has been highly dependant on natural resources for centuries [16]. Other than that, it will also benefit on air pollution detection that give negative impact to health [17].

Figure 1 demonstrate the unmixing process that involving four main steps that are atmospheric correction, dimensionality reduction, unmixing, and inversion [18]. The first step is considering the atmospheric correction of the radiance data cube. The second step is data reduction after converting the property of data cube. The third step is unmixing, and the last step is inversion. Unmixing which may be tackled via the classical endmember determination plus inversion or via sparse regression or sparse coding approaches.

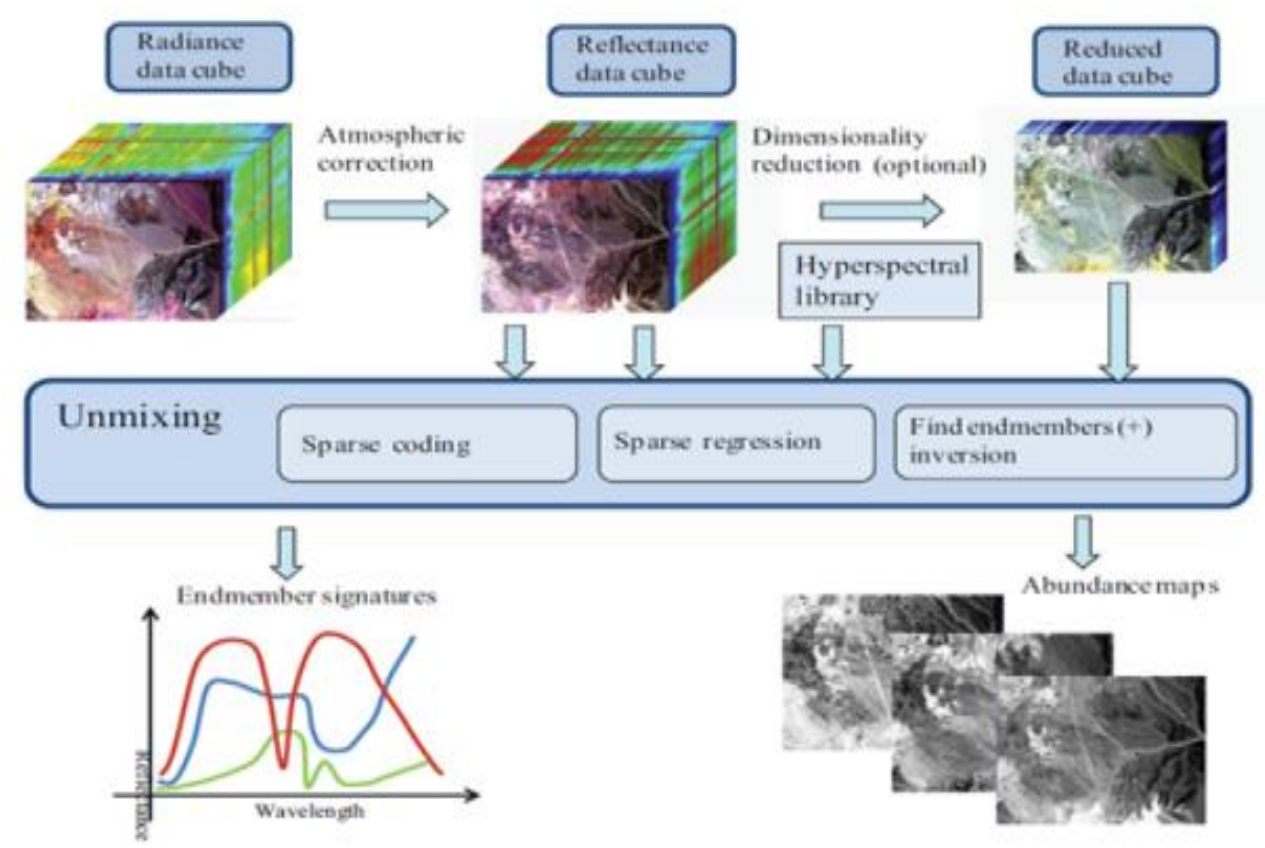

Figure 1. The steps of unmixing process [18]

\section{RESEARCH METHOD}

Systematic models for the mixing of various materials provide the foundation for developing techniques to recover estimates of the constituent substance spectra and their proportions from mixed pixels. An entire model of the mixing procedure, in any case, is more muddled than a basic depiction of how surface mixing connect. Mixing models can likewise consolidate the impacts of the three-dimensional topology of items in a scene, for example, the tallness of trees, the size and thickness of their overhangs, and the sensor perception edge. The essential introduce of blend displaying is that inside a given scene, the surface is overwhelmed by few particular materials that have moderately consistent spectral properties. These particular substances (e.g., water, grass, mineral composes) are called endmembers, and the portions in which they show up in a blended pixel are called fragmentary plenitudes. On the off chance that the vast majority of the spectral fluctuation inside a scene is a result of endmembers showing up in differing extents, it legitimately takes after that some mix of their unearthly properties can demonstrate the otherworldly inconstancy saw by the remote detecting framework [19]. 
Figure 2 illustrates how linear mixing radiation reflects from the surface. The way of the reflection in linear surface is portrayed as a checkerboard mixture and any given package of incident radiation only interact with one component for example no multiple scattering between components [20]. If the total surface area is considered to be divided proportionally according to the fractional abundances of the endmembers, then the reflected radiation will convey the characteristics of the associated media with the same proportions. In this sense, there exists a linear relationship between the fractional abundance of the substances comprising the area being imaged and the spectra in the reflected radiation [21].

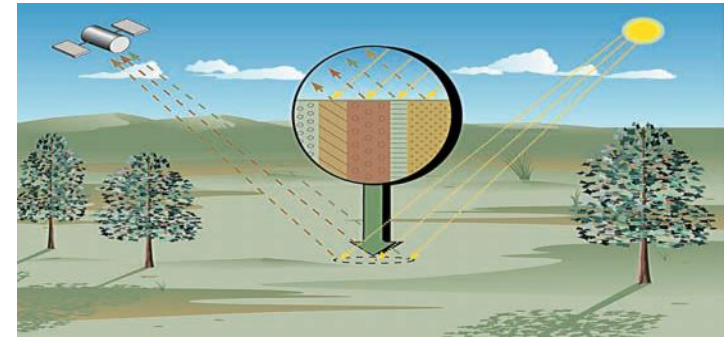

Figure 2. Illustration of linear mixing radiation reflects from surface [20].

\subsection{Algorithms for hyperspectral unmixing}

The resolution cell corresponding to a single pixel in an image contains several substances. In this situation, the scattered energy is a mixing of the endmember spectra [19,22]. A challenging task underlying many hyperspectral imagery applications is then decomposing a mixed pixel into a collection of reflection spectra called endmember signatures. It also true that it is not exempt or drawbacks, such as the fact that all the possible combinations of algorithms in order to fully unmixing a hyperspectral image according to the aforementioned processing chain demand a formidable computational effort, which tend to be higher the better the performance of the designed unmixing chain is. The algorithms exploit two facts: 1) the endmember are the vertices of a simplex and 2) the affine transformation of a simplex is also a simplex [23].

\subsection{N-FINDR algorithms}

One of the earliest approaches is the N-FINDR algorithms is a selection algorithms. Its work is described as follow: it starts with a random collection of image pixel spectra, corresponding to the initial set of endmembers [24]. Then, each of the remaining image pixels is considered as a candidate to replace each endmembers, if doing so the volume of the simplex increases, then it is accepted. The N-FINDR algorithms require a dimension reduction step, originally an orthogonal subspace projection (OSP) to a space of dimension $\mathrm{N}-1$, where $\mathrm{N}$ is the number of endmember [25, 26]. This set of endmembers found by the N-FINDR would not allow the nonnegative unmixing of the pixel spectra in general [7].

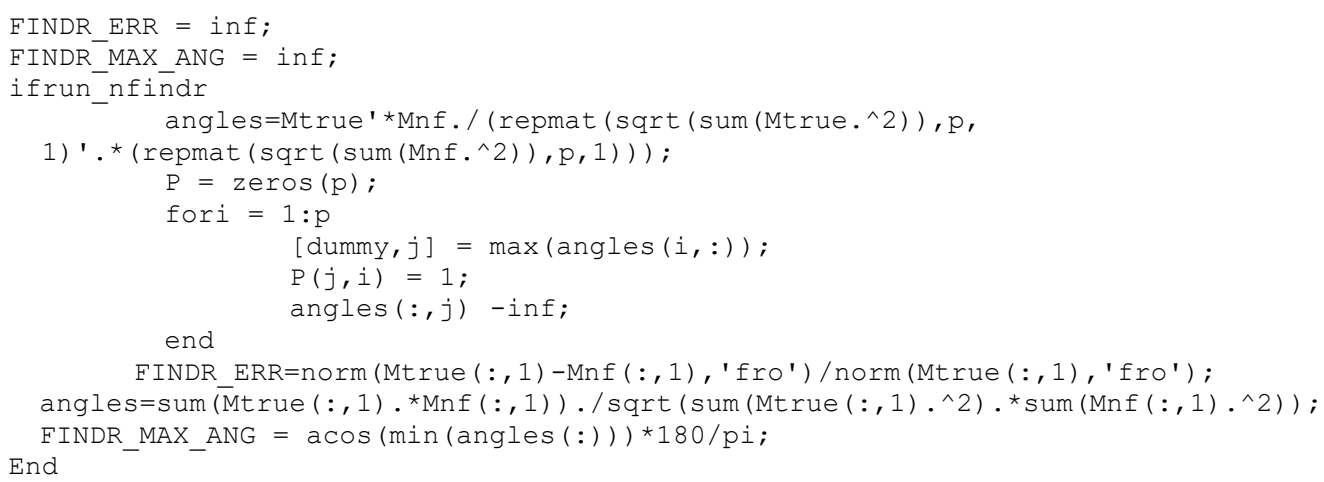

\subsection{VCA algorithms}

The vertex component analysis algorithms (VCA) are presented in this hyperspectral [8, 9]. This algorithm is unsupervised and exploits that the affine transformation of a simplex is also simplex. It works with projected and unprojected data [26, 27]. The algorithms iteratively projects data onto a direction orthogonal to the subspace spanned by the endmembers already determined. The new endmembers signature corresponds to the extreme of the projection. The algorithms iterates until all endmembers are exhausted. 


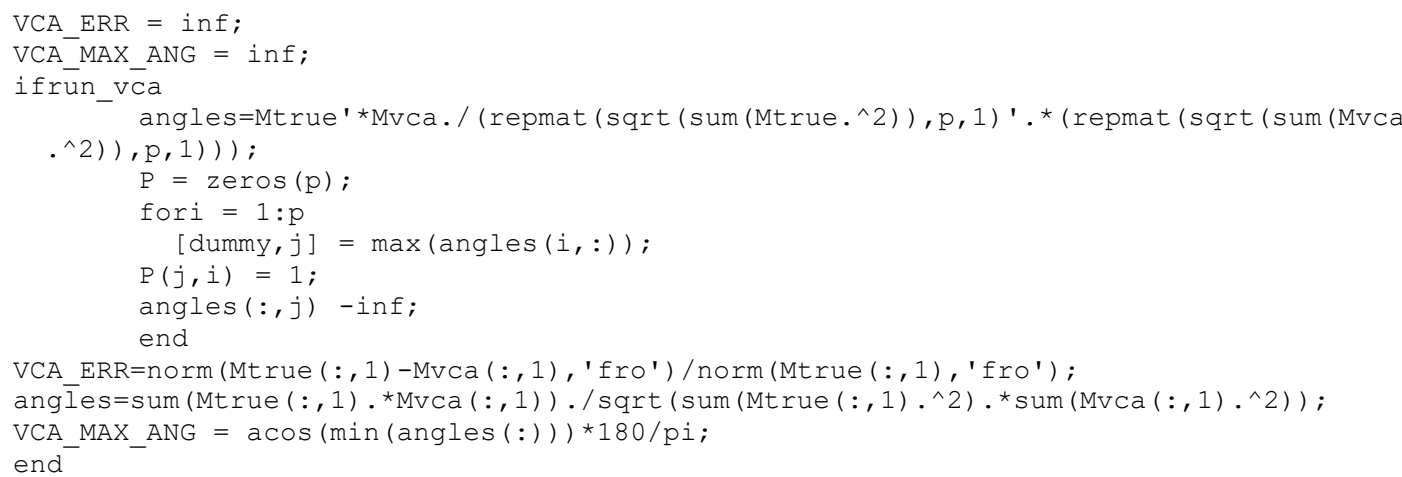

\subsection{SISAL algorithm}

The SISAL (simple identification via variable splitting and augmented Lagrangian) algorithm also able performs the endmember extraction for hyperspectral unmixing. The features of these algorithms are implementing a robust version of the minimum volume concept $[10,11]$. It robustness allowing the positivity constrains to be violated in order to estimate endmember more precise. The noise vector is considered. The existing of noise or any other source, the spectral vectors may lie outside the true data simplex [28].

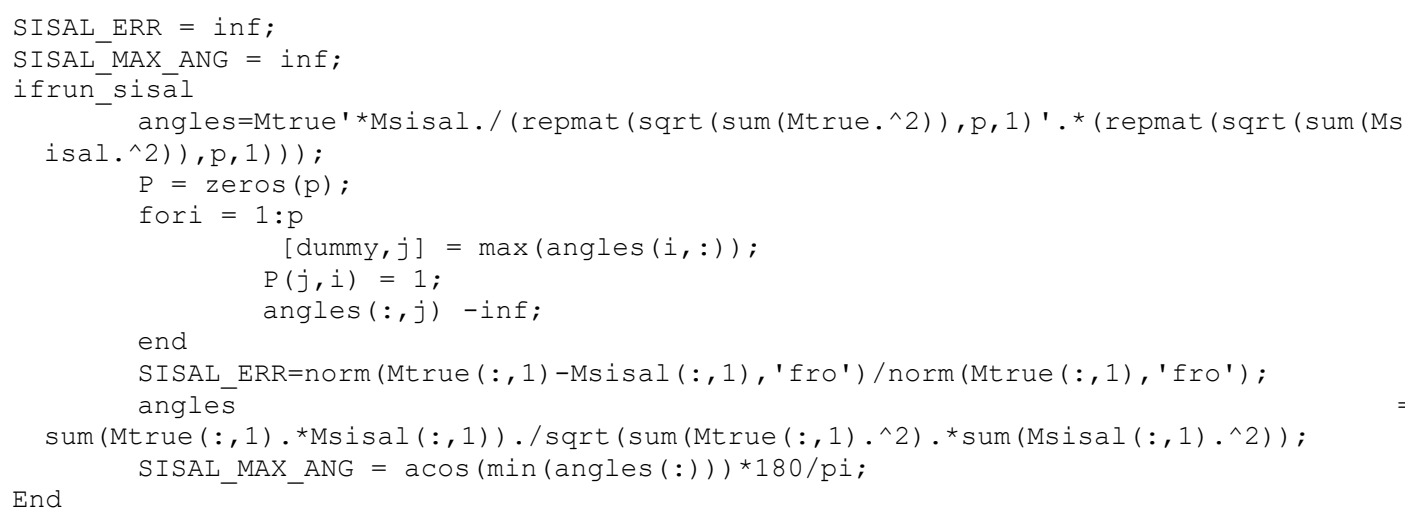

\subsection{CoNMF algorithm}

CoNMF is used with another regularization term to promote minimum volume by pushing the endmembers toward the mean value of the data set or bring the endmembers to the real solution quadratically regularized by a given simplex, other than the minimum volume $[12,13]$. CoNMF does not require a prior step to estimate the number of endmembers and can be effectively used in scenarios in which the number of endmembers is not known a priori [29].

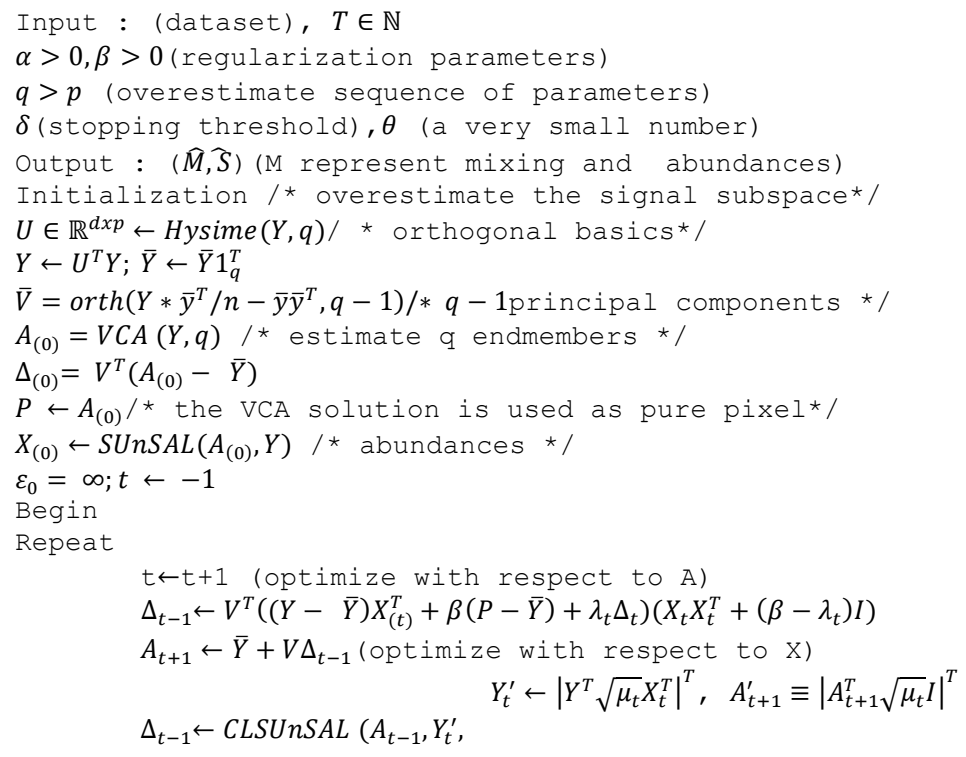




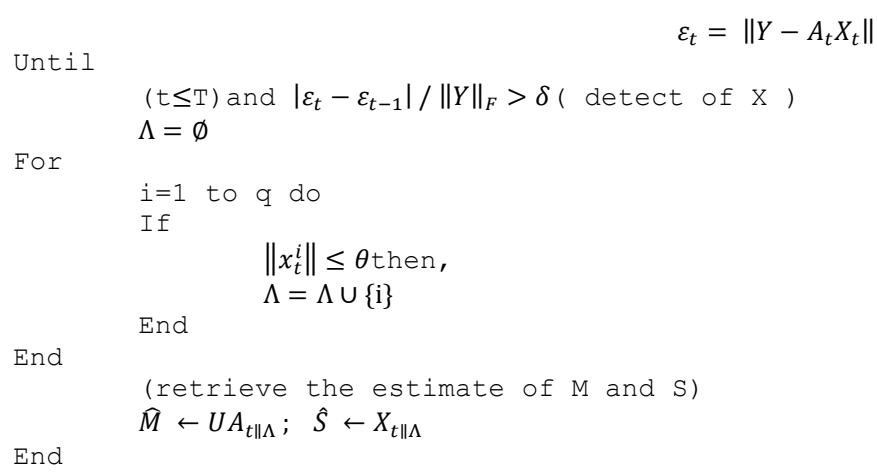

In this paper, the USGS library for mineral is used as ground truth for real endmember that contain 5 endmembers as reference. The database for algorithm is taken from previous paper [7, 8, 10, 12]. It is then generated in Matlab 2019 software on Acer Swift SF314-41 with AMD Ryzen 5 3500U with Radeon Vega Mobile Gfx 2.10GHz processor, 64-bit operating system type, with 8Gb RAM.

\section{RESULTS AND ANALYSIS}

The SAD is used to evaluate the performance of estimated endmembers, which is an angle distance between an estimated endmember and its corresponding ground truth. It defined as:

$$
\operatorname{SAD}(\mathrm{m}, \dot{\mathrm{m}})=\arccos \left(\frac{\mathrm{m}^{\mathrm{T}} \dot{\mathrm{m}}}{\|\mathrm{m}\| \cdot\|\dot{\mathrm{m}}\|}\right)
$$

where $m$ denotes the ground truth of one endmember, while $\dot{m}$ represent the corresponding estimated results. The smaller SAD corresponds to a better performance. One of the main aspects in this project is the unmixing process of the proposed compared algorithms. Figure 3 show the comparison of five endmember of spectral signature with respect to the endmember extraction by four algorithms. The material for these five endmember is tabulated in Table 1. From Figure 3, the dotted line is true spectral signature from library spectral compared purple line extracted by CoNMF algorithm, red line is extracted by NFINDR algorithm, green line is extracted by VCA algorithm and the last one blue line is extracted by SISAL algorithm. The entire algorithm using same data but different technique of extraction of endmembers. Hence, all algorithm demonstrates excellent extraction by resulting the spectral signature almost exactly same with spectral library.
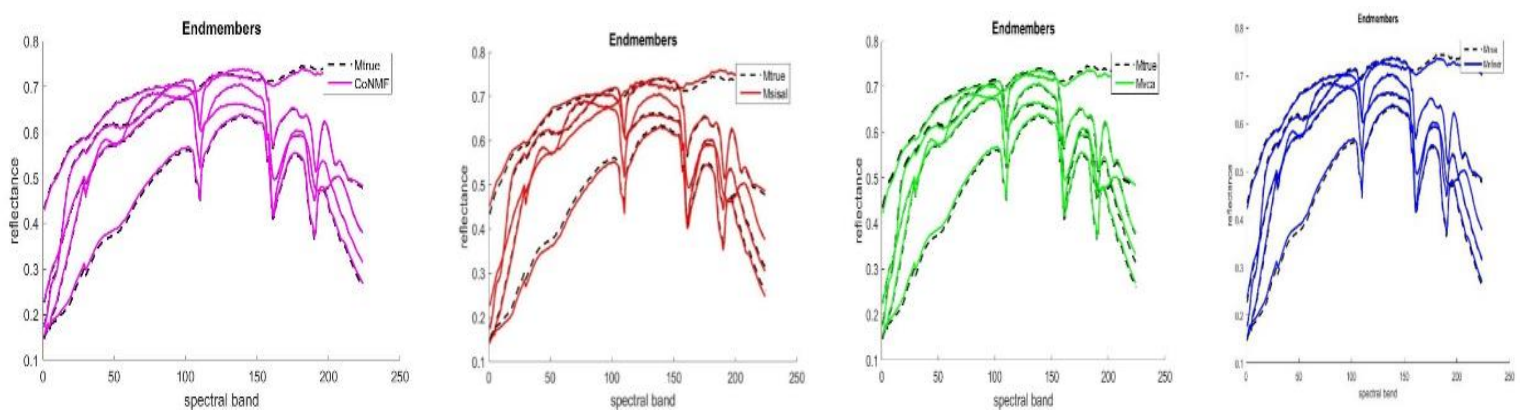

Figure 3. Comparison of the five spectral signatures in cuprite scene with different algorithms.

Table 1. Reference spectral signature

\begin{tabular}{ll}
\hline Endmember & Name of material \\
\hline Endmember 1 & \#6 kaolinite \\
Endmember 2 & \#10 pyrope \\
Endmember 3 & \#8 montmorillonite \\
Endmember 4 & $\# 5$ kaolinite \\
Endmember 5 & $\# 12$ chalcedony \\
\hline
\end{tabular}


The first experiment aims to investigate the best spectral angle distance estimated by four difference algorithms. Table 2 point out the difference performance of spectral angle distance estimated by four difference algorithms with difference noise level. The first column of table show the noise level that was used. The four left columns list the spectral angle distance difference between estimated difference algorithms. At noise level is $30 \mathrm{db}$, the SAD for CoNMF is 0.619155 , SAD for VCA is 0.890067 , SAD for NFINDR is 0.847781 and SAD for SISAL is 2.568448, which is CoNMF algorithm is smaller than other and show best performance. But, it difference with using noise level $40 \mathrm{db}$, the value SAD for all algorithms drop more smaller than using $30 \mathrm{db}$. The SAD for CoNMF is 0.495250 , SAD for VCA and NFINDR is 0.552555 , and SAD for SISAL is 0.727632 . The third noise level is $60 \mathrm{db}$, The SAD for CoNMF is 0.552818 , SAD for VCA and NFINDR is 0.659840 , and SAD for SISAL is 0.023277.

Table 2. SAD and different noise level with SNR value $30 \mathrm{~dB}, 40 \mathrm{~dB}, 60 \mathrm{~dB}$

\begin{tabular}{lllll}
\hline \multirow{2}{*}{ SNR } & \multicolumn{4}{l}{ Algorithm (SAD) } \\
& CoNMF & VCA & NFINDR & SISAL \\
\hline 30 & 0.619155 & 0.890067 & 0.847781 & 2.568448 \\
40 & 0.495250 & 0.552555 & 0.552555 & 0.727632 \\
60 & 0.552818 & 0.659840 & 0.659840 & 0.023277 \\
\hline
\end{tabular}

The second experiment aims to measure the spectral angle distance for each endmember by the noise level for $30 \mathrm{db}$ and $40 \mathrm{~dB}$. Table 3 shows the result of the experiment. The results proves that the SAD for endmember 1 extracted by CoNMF algorithms is archived $100 \%$ similar with the original endmember. Besides, the SAD for endmember 3 extracted by SISAL algorithm show smaller than extracted CoNMF algorithm. If the smaller SAD, better performance of unmixing process.

Table 3. SAD between five endmember for SNR 30dB and 40dB

\begin{tabular}{llllllllll}
\hline \multirow{2}{*}{ SNR (30) } & \multicolumn{3}{c}{ Algorithm (SAD) } & \multicolumn{3}{c}{ Algorithm (SAD) } \\
& CoNMF & VCA & NFINDR & SISAL & SNR (40) & CoNMF & VCA & NFINDR & SISAL \\
\hline Endmember 1 & 0.000000 & 0.356500 & 0.356500 & 1.127762 & Endmember 1 & 0.000000 & 0.476512 & 0.324057 & 0.290746 \\
Endmember 2 & 0.404453 & 0.802739 & 0.802739 & 2.072371 & Endmember 2 & 0.438316 & 0.530236 & 0.530236 & 0.631297 \\
Endmember 3 & 0.493473 & 0.646359 & 0.231876 & 0.231876 & Endmember 3 & 0.249545 & 0.331000 & 0.331000 & 0.183731 \\
Endmember 4 & 0.619155 & 0.839424 & 0.847781 & 2.568448 & Endmember 4 & 0.495250 & 0.552555 & 0.552555 & 0.727632 \\
Endmember 5 & 0.180157 & 1.633885 & 0.506502 & 0.890067 & Endmember 5 & 0.188265 & 0.337724 & 0.337724 & 0.540945 \\
\hline
\end{tabular}

The next experiment aims to measure the spectral angle distance for each endmember by the noise level $60 \mathrm{~dB}$. Table 4 shows the result of the experiment. The results proves that the SAD for endmember 1 extracted by CoNMF algorithms is archived $100 \%$ similar with the original endmember and extracted by SISAL algorithm exactly similar to the real ones. Besides, the SAD for endmember 2 and 3 extracted by SISAL algorithm show smaller than extracted CoNMF algorithm. But the SAD for endmember 4 and 5 is extracted by VCA algorithm show smaller other and better performance than other algorithms. If the smaller SAD, better performance of unmixing process.

For illustrative purpose, Figure 4 show the comparison of the real endmember with estimate endmember extracted with different algorithm but resulting same spectral signature. The SNR used is $40 \mathrm{~dB}$ as it shows the best result compared to the other algorithms. This estimation obtained on real data in absence of pure pixel. The SAD for endmember is calculated based on estimated endmember and true endmember. The result shows the estimated signature by CoNMF are similar to the real ones, whereas the other algorithm is slightly different. This experiment show that CoNMF is the best algorithm to be used in hyperspectral unmixing other than the other three algorithms.

Table 4. SAD between five endmember for SNR 60dB

\begin{tabular}{lllll}
\hline \multirow{2}{*}{ SNR (60) } & \multicolumn{4}{l}{ Algorithm (SAD) } \\
CoNMF & VCA & NFINDR & SISAL \\
\hline Endmember 1 & 0.000000 & 0.588549 & 0.588549 & 0.002067 \\
Endmember 2 & 0.326088 & 0.533598 & 0.533598 & 0.023277 \\
Endmember 3 & 0.198221 & 0.210019 & 0.210019 & 0.004687 \\
Endmember 4 & 0.552818 & 0.013281 & 0.598216 & 0.598216 \\
Endmember 5 & 0.506061 & 0.011777 & 0.659840 & 0.659840 \\
\hline
\end{tabular}

Indonesian J Elec Eng \& Comp Sci, Vol. 20, No. 2, November 2020 : 813 - 821 

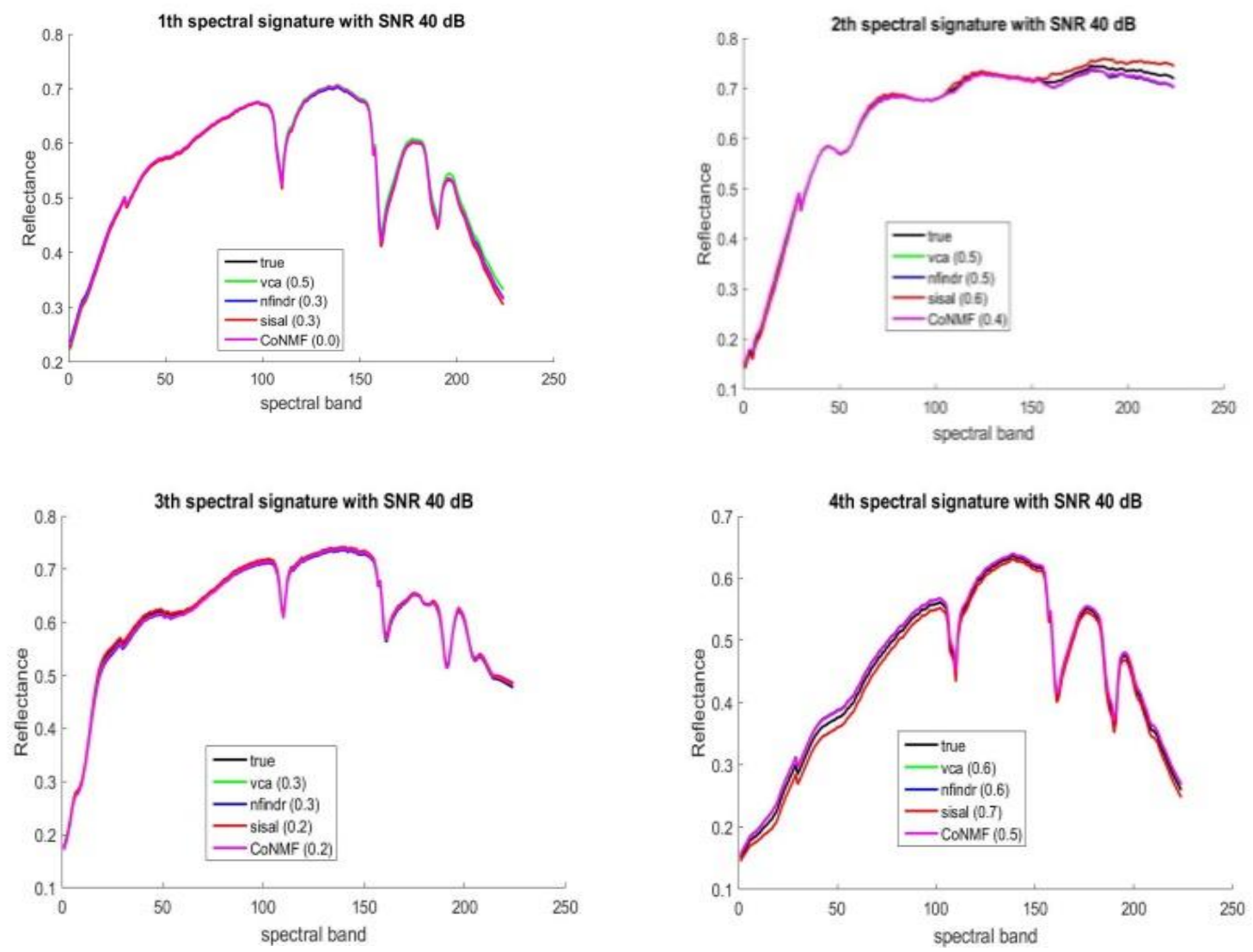

Figure 4. Comparison each endmember for different algorithm with snr $40 \mathrm{~dB}$

\section{CONCLUSION}

Four major algorithms on unmixing approaches CoNMF, VCA, NFINDR, and SISAL have been studied. The results are compared between each algorithms based on the SAD value of spectral signature. Under different noise levels, when the noise level is moderately low at $40 \mathrm{~dB}$, the algorithms can obtain very good unmixing results. In turn, when SNR larger than or smaller than $40 \mathrm{~dB}$, the quality of the unmixing result decrease particularly regard to the SAD value. However, the SNR start with $30 \mathrm{~dB}$ because $20 \mathrm{~dB}$ is not realistic noise level for imaging spectrometer. As a result, it can conclude that $40 \mathrm{~dB}$ indicate very high robustness to noise by algorithms, and CoNMF is the best algorithm to be used on unmixing approach compared to the other algorithms.

\section{ACKNOWLEDGEMENTS}

The author would like to thanks to the Ministry of Education (MOE) for sponsoring this work under project (FRGS/2017/TK04/UTEM/02/18) and CeTRI, Faculty of Electronics and Computer Engineering (FKEKK), UniversitiTeknikal Malaysia Melaka (UTeM) for the moral support throughout the project.

\section{REFERENCES}

[1] N. Keshava and J. F. Mustard, "Spectral unmixing," IEEE Signal Process. Mag., vol. 19, no. 1, pp. 44-57, 2002.

[2] J. M. Bioucas-Dias et al., "Hyperspectral unmixing overview: Geometrical, statistical, and sparse regression-based approaches," IEEE J. Sel. Top. Appl. Earth Obs. Remote Sens., vol. 5, no. 2, pp. 354-379, 2012.

[3] J. M. Bioucas-Dias and J. M. P. Nascimento, "Hyperspectral subspace identification," IEEE Trans. Geosci. Remote Sens., vol. 46, no. 8, pp. 2435-2445, Aug. 2008.

[4] J. Li, J. M. Bioucas-Dias, A. Plaza, L. Liu, "Robust collaborative nonnegative matrix factorization for hyperspectral unmixing," IEEE Transactions on Geoscience and Remote Sensing, Vol. 54, no. 10, pp. 6076-6090, 2016.

[5] M-D. Iordache, et al, "A dynamic unmixing framework for plant production system monitoring," IEEE Journal of Selected Topics in Applied Earth Observations and Remote Sensing, Vol. 7, no. 6, pp. 2016-2034, 2014.

[6] A. Plaza, Q. Du, J. Bioucas-Dias, X. Jia, and F. Kruse, "Foreword to thespecial issue on spectral unmixing of remotely sensed data," IEEETrans.Geosci. Remote Sens., vol. 49, no. 11, pp. 4103-4110, Nov. 2011. 
[7] S. W. Dowler, R. Takashima, M. Andrews, "Reducing the complexity of the N-FINDR algorithm for hyperspectral image analysis," IEEE Transactions on Image Processing, vol. 22, no. 7, pp. 2835 - 2848, 2013.

[8] J. M. P. Nascimento, J. M. Bioucas-Dias, "Vertex component analysis: A fast algorithm to unmix hyperspectral data," IEEE Transactions on Geoscience and Remote Sensing, vol. 43, no. 4, pp. 898-910, 2005.

[9] C-I. Chang, S-Y. Chen, H-C. Li, H-M. Chen, C-H. Wen, "Comparative study and analysis among ATGP, VCA, and SGA for finding endmembers in hyperspectral imagery," IEEE Journal of Selected Topics in Applied Earth Observations and Remote Sensing, vol. 9, no. 9, pp. 1-27, 2016.

[10] J. Sevilla, G. Martín, and J. M. P. Nascimento, "Parallel hyperspectral unmixing method via split augmented lagrangian on GPU," IEEE Geoscience and Remote Sensing Letters, vol. 13, no. 5, pp. 626-630, 2016.

[11] J. M. Bioucas-Dias, "A variable splitting augmented lagrangian approach to linear spectral unmixing," First Workshop on Hyperspectral Image and Signal Processing: Evolution in Remote Sensing, pp. 1-4, 2009.

[12] C. Févotte and N. Dobigeon, "Nonlinear hyperspectral unmixing with robust nonnegative matrix factorization," IEEE Trans. Image Process., vol. 24, no. 12, pp. 4810-4819, 2015.

[13] R. Rajabi, H. Ghassemian, M. Khodadadzadeh, "Graph regularized nonnegative matrix factorization for hyperspectral data unmixing," 2011 7th Iranian conference on machine vision and image processing, pp. 1-4, 2011.

[14] C. Andreou, V. Karathanassi, "A novel multiple endmember spectral mixture analysis using spectral angle distance," IEEE International Geoscience and Remote Sensing Symposium, pp. 4110-4113, 2012

[15] R. Rajabi, H. Ghassemian, "Hyperspectral data unmixing using GNMF method and sparseness constraint," IEEE International Geoscience and Remote Sensing Symposium, pp. 1450-1453, 2013.

[16] A. D. Mengistu, D. M. Alemayehu, "Soil characterization and classification: A hybrid approach of computer vison and sensor network," International Journal of Electrical and Computer Engineering (IJECE), vol. 8, No. 2, pp. 989-995, April 2018.

[17] S. R. Sulistiyanti, F. X. A. Setyawan, M. Komarudin, "Detection air pollution based on infrared image processing," Telkomnika, vol. 17, No. 4, pp. 1796-1802, 2019.

[18] G. Zhang, Y. Xu, F. Fang, "Framelet-Based Sparse Unmixing of Hyperspectral Images." IEEE Transactions on Image Processing, vol. 25, no. 4, pp 1-13, 2016.

[19] L. Zhuang, B. Zhang, L. Gao, J. Li, and A. Plaza, "Normal endmember spectral unmixing method for hyperspectral imagery," IEEE J. Sel. Top. Appl. Earth Obs. Remote Sens., vol. 8, no. 6, pp. 2598-2606, 2015.

[20] W-K Ma, et al, C-Y. Chi, "A signal processing perspective on hyperspectral unmixing," IEEE Signal Processing Magazine, vol. 31, no. 1, pp. 67-81, 2014.

[21] Y. Zhang, D. Wang, L. Kan, P. Zhao, "A study on image reconfiguration algorithm of compressed sensing," Telkomnika, vol. 15, no. 1, pp. 299-305, March 2017.

[22] F. Feng, C. Deng, W. Wang, J. Dai, Z. Li, B. Zhao, "Constrained Nonnegative Matrix Factorization for Robust Hyperspectral Unmixing," IEEE International Geoscience and Remote Sensing Symposium, pp. 4221-4224, 2018.

[23] Z. Shi, W. Tang, Z. Duren, Z. Jiang, "Subspace matching pursuit for sparse unmixing of hyperspectral data," IEEE Transactions on Geoscience and Remote Sensing, vol. 52, no. 6, pp. 3256-3274, June 2014.

[24] C-I. Chang, C-C. Wu, C-T. Tsai, "Random N-Finder (N-FINDR) endmember extraction algorithms for hyperspectral imagery," IEEE Transactions on Image Processing, vol. 20, no. 3, pp. 641-656, March 2010.

[25] X. Zhang, X-H. Tong, M-L Liu, "An improved N-FINDR algorithm for endmember extraction in hyperspectral imagery," Joint Urban Remote Sensing Event, pp. 1-5, 2009.

[26] Y. Kim, Y. Kim, "Hyperspectral image classification based on spectral mixture analysis for crop type determination," IEEE International Geoscience and Remote Sensing Symposium, pp. 5304-5307, 2018.

[27] N. Patel, H. Soni, "Anomaly Detection using VCA Algorithm for Multi-Temporal Hyperspectral Images," International Conference on Wireless Communications, Signal Processing and Networking (WiSPNET), pp. 2248-2252, 2017.

[28] Y. Itoh, A. Iwasaki, "Enhancement of hyperspectral unmixing using continuum removal," IEEE International Geoscience and Remote Sensing Symposium-IGARSS, pp. 445-448, 2013

[29] Y. Wu, B. Shen, H. Ling, "Visual tracking via online nonnegative matrix factorization," IEEE Transactions on Circuits and Systems for Video Technology, vol. 24, no. 3, pp. 374-383, 2013.

\section{BIOGRAPHIES OF AUTHORS}

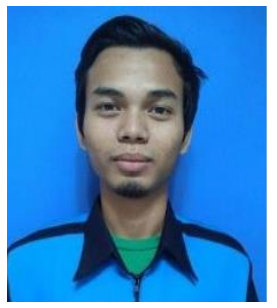

Ain Zat Mohd Yusof received his Bachelor's degree in Electronic and Computer engineering from Technical University of Malaysia Malacca in 2016. Since 2018, he has been pursuing his Master's degree in the same university, majoring in Signal Processing. His research interest include multispectral imaging, hyperspectral imaging, and spatial and spectral constraint. 


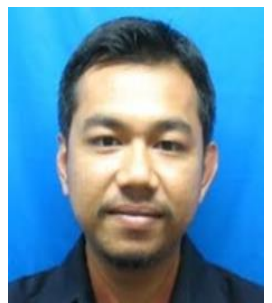

Redzuan Abdul Manap received the BEng degree in electrical and electronic engineering from The University of Nottingham, UK in 2002, the MSc degree in communication and signal processing from University of Newcastle upon Tyne, UK in 2004 and the $\mathrm{PhD}$ degree in electronic engineering from The University of Sheffield, UK in 2018. He is currently a Senior Lecturer at Faculty of Electronics and Computer Engineering, Universiti Teknikal Malaysia Melaka (UTeM), Malaysia. His research interests include image processing, visual perception, machine learning and computer vision. He has published around 20 journals and proceeding papers in these areas.

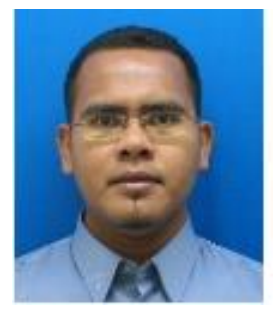

Abd Majid Darsono was born in Malaysia in 1980. He received the Bachelor degree in communication and computer engineering from Universiti Kebangsaan Malaysia in 2002 and the MSc degree in electronic communications and computer engineering from The University of Nottingham, United Kingdom in 2005. In 2012, he received the Ph.D. degree in signal processing from University of Newcastle upon Tyne, United Kingdom. He joined Universiti Teknikal Malaysia Melaka (UTeM) since 2005 as a lecturer. He is currently an Associate Professor in Faculty of Electronics and Computer Engineering in UTeM. His research experience and interests include statistical signal processing, and speech and image processing. 